\title{
A Retrospective Analysis of Hospital Electrocardiogram Auto-Populated QT Interval Calculation
}

\author{
Adam L. Rosenblum ${ }^{1,2}$, Ariana C. Dremonas ${ }^{1,2}$, Scott C. Stockholm 1, 2 , Nicholas L. Biondi ${ }^{1,2}$ \\ 1. Internal Medicine, Cape Fear Valley Health System, Fayetteville, USA 2. Internal Medicine, Campbell University \\ School of Osteopathic Medicine, Buies Creek, USA
}

Corresponding author: Adam L. Rosenblum, humblefish@gmail.com

\section{Abstract \\ Background}

The current electrocardiogram (ECG) standard for rate correction of the QT interval (QTc) is a power function known as the Bazett formula (QTcB). QTc formulae are either power functions or linear functions. QTcB is known to lack reliability, as heart rate (HR) rises from or falls below 60 beats per minute (bpm). The American Heart Association (AHA), the American College of Cardiology Foundation (ACCF), and the Heart Rhythm Society (HRS) have recommended using other formulae in place of QTcB since 2009. The Epic Electronic Health Record System (Epic Systems Corporation, Verona, WI) automatically populates the Fridericia formula (QTcFri) on hospital ECG reports without any provider calculation.

\section{Methods}

We aimed to retrospectively investigate the effect of QTcFri on one year of ECGs in the Epic Electronic Health Record (EHR) at a single tertiary care center. Inclusion criteria for ECG reports specified HR 60-120 bpm without QRS duration > 120 ms. Gathered data from Epic EHR ECG reports included patient age, sex, HR, QRS duration (QRSd), QT interval, QTcB, and QTcFri. EHR documented 61,946 ECG reports for the year, with 44,566 meeting criteria for inclusion. General statistical methods included range, median, mean, and standard deviation. Confidence intervals were assessed to maintain the fidelity of analysis. The normality of data distribution was assessed with Kolmogorov-Smirnov testing. The Wilcoxon rank-sum test was then performed to confirm a statistically significant difference between the Bazett and Fridericia formulae. The $\triangle$ QTc analysis was conducted on prolonged QTc (males $>450 \mathrm{~ms}$; females $>460 \mathrm{~ms}$ ) and severely prolonged QTc $>500$ ms data subsets. A value of $\mathrm{p}<0.05$ was interpreted as significant. Statistical analysis was performed using SPSS statistical software (IBM Statistics, v. 26; IBM Corp, Armonk, NY).

\section{Results}

The 44,566 ECG reports demonstrated $57 \%$ female gender and a mean age of $57 \pm 17.5$ years. The mean HR was $83 \pm 14.7 \mathrm{bpm}$ and the mean $\triangle \mathrm{QTc}$ was $23 \pm 12.9 \mathrm{~ms}$ shorter with QTcFri. Mean data showed minimal variation between sexes: age, heart rate, uncorrected QT, QTcB, QTcFri, and $\triangle$ QTc varied by less than $2 \%$. Mean QRS varied by $4 \%$ between sexes. The Wilcoxon rank-sum test revealed 44,127 ranks with a negative difference, 0 ranks with a positive difference, and 439 ties, p <0.001 ( $99 \%$ CI: $22.5 \mathrm{~ms}, 23.0 \mathrm{~ms}$ ). QTcB identified 37.4\% (16665/44566) ECGs prolonged. Using QTcFri, 21\% (9371/44566) of the total ECGs corrected to normal QTc (<450 ms (men) and $460 \mathrm{~ms}$ (women)). QTcFri use reduced the number of ECG reports with QTC $>500 \mathrm{~ms}$ by $57.3 \%$. A total of $125 \mathrm{ECG}$ reports, 117 females and eight males, corrected to normal genderspecific QTc with QTcFri. The mean decrease in QTc with the Fridericia formula when QTcB > $500 \mathrm{~ms}$ was 31 $\pm 14.5 \mathrm{~ms}$ ( $99 \%$ CI: $30.4 \mathrm{~ms}, 31.7 \mathrm{~ms}$ ).

\section{Conclusion}

Our data from the Wilcoxon rank-sum analysis indicated that the EHR QTcFri analysis yields a statistically significant difference $(\mathrm{p}<0.001)$ in QTc calculation of $22 \mathrm{~ms}$ over 44,566 ECG reports. The data showed a $21 \%$ reduction in inaccurately documented test results. The utilization of this resource will provide the most accurate and clinically relevant data to inform clinical decision-making. Accurate QT interval calculation will better inform downstream clinical decision-making through a wider scope of therapeutic intervention. This analysis is readily available to clinicians without calculation and its awareness will benefit patient care.

Categories: Cardiology, Emergency Medicine, Internal Medicine

Keywords: qt interval, qt calculation, 12-lead, electrocardiogram (ecg/ekg), torsades de pointes (tdp)

\section{Introduction}

The QT interval of an electrocardiogram (ECG) was originally named by Willem Einthoven in 1895 in accordance with the mathematical tradition established by Descartes [1]. The interval corresponds to the 
electrical systole of the cardiac cycle - the time duration spanning from the opening of fast $\mathrm{Na}^{+}$and L-type

$\mathrm{Ca}^{++}$ion channels, initiating Phase 0 of ventricular depolarization, until the closing of inward-rectifier $\mathrm{K}_{1}$ current dependent channels, terminating phase 4 ventricular repolarization. The magnitude of the interval is inversely proportional to the heart rate (HR): increasing or decreasing heart rate will respectively contract or expand the time interval between beats, affecting the QT interval on ECG.

Dr. Henry Cuthbert Bazett evaluated the ECGs of 39 people in 1920 to derive the current standard QT interval rate-correction (QTc) formula, used as a surrogate evaluation of mechanical systole [2]. Since Bazett's analysis, numerous approaches with either power functions or linear functions, each with inherent idiosyncrasies, have been proposed. Among these formulaic curiosities is a consistent observation that Bazett's calculation (QTcB) produces increasing QTc inaccuracy, as the heart rate rises in excess or falls below a rate of 60 beats per minute (bpm) [3].

Evidence collected since the 1980s prompted the American Heart Association (AHA), the American College of Cardiology Foundation (ACCF), and the Heart Rhythm Society (HRS) to issue guidelines on appropriate ECG interpretation in 2009, recommending against the use power-function formulae [3]. The unreliability of QTcB at HR above or below 60 bpm continued to be corroborated in research following the 2009 Guidelines, and the recommendation for alternative formula use over QTcB was reaffirmed by the 2017 updated AHA Scientific Statement [4].

One of the greatest barriers to changing the ECG standard from QTcB to another QTc formula has been the lack of consensus evidence regarding which alternate formula demonstrates clinical superiority. Retrospective research from 2016 demonstrated that the Framingham (QTcFra) and Fridericia (QTcFri) formulae exhibit superiority to the Bazett Formula at heart rates less than 90 beats per minute [5]. QTcFra outperformed the Hodges formula (QTcH), the Rautaharju formula (QTcR), QTcB, and QTcFri with regard to both 30-day and one-year mortality - however, no statistically significant distinction between QTcFra and QTcFri was determined for either metric [5]. Rautaharju formula (QTcR), a linear-function formula, was studied in 57,595 subjects and is specific to both gender and QRS duration $[2,6]$. Currently, no prospective data exist to corroborate these retrospective analyses and identify which formula is to replace QTcB. Guidelines continue to recommend a specific methodology over a specific formula: linear-function formulae (i.e. QTcH, QTcR) use is supported by computational model evidence over power-function formulae use (i.e. QTcB, QTcFri, QTcFra) [2-3]. The guidelines further recommend formula citation with each interpretation in an effort to avoid confusion between ECG documented QTcB and any other corrected QT formula [3].

Accurate QTc analysis is vitally important to patient care. QTc use is integrated into the clinical decisionmaking process for both drug class and frequency of medication administration - due to the common offtarget side effect of many medications to prolong the QT interval. Additionally, using more accurate QTC calculation is essential for monitoring the risk of ventricular arrhythmia e.g. Torsades de Pointes (TdP). Normal QTc is gender-dependent, with male values ranging from $350-450 \mathrm{~ms}$ and female values ranging from 350-460 ms [3]. The risk for TdP increases as QTcB increases at values greater than $500 \mathrm{~ms}$ [7-8]. QT interval prolonging medications are normally held as QTcB approaches $500 \mathrm{~ms}$ - a formula-dependent threshold. Increasing provider awareness of available QTc methods has the potential to directly impact patient care via decreasing the above metrics by using guideline-based formulae.

\section{Materials And Methods}

\section{Study design}

This single-center, retrospective, non-randomized, non-blinded, observational study examined the use and diagnostic impact of two Electronic Health Record (EHR) auto-populated QTc values on ECG reports, QTcB and QTcFri. ECG reports were collected from May 2019 to April 2020 at Cape Fear Valley Medical Center - a suburban, tertiary care center in Fayetteville, North Carolina. ECGs were recorded using Phillips IntelliSpace ECG devices (Phillips Healthcare Solutions, Franklin, TN). Standard 12-lead ECGs were collected with $25 \mathrm{~mm} / \mathrm{s}$ paper speed, $10 \mathrm{~mm} / 1 \mathrm{mV}$ amplitude, and $250 \mathrm{~Hz}$ sampling rate. Gathered data from Epic EHR ECG reports (Epic Systems Corporation, Verona, WI) included patient age, sex, HR, QRS duration (QRSd), QT interval, QTcB, and QTcFri. The study was approved by the Cape Fear Valley Health Internal Review Board and data were collected retrospectively and deidentified.

\section{Patient population}

ECG reports were obtained in the hospital from both male and female patients aging from 18 to 105 years. ECG reports were assessed for a minimum heart rate of $60 \mathrm{bpm}$, a maximum heart rate of $120 \mathrm{bpm}$, and a QRS duration of less than $120 \mathrm{~ms}$. ECG report data were inspected for quality and excluded from the analysis if $\mathrm{HR}<60 \mathrm{bpm}$ or $>120 \mathrm{bpm}$, QRS > $120 \mathrm{~ms}$, missing EHR parameters, or if erroneous in the extreme (e.g. age 120 years).

The study was designed with the intent of applying minimal exclusion criteria to maximize the study sample size while ensuring the fidelity of the data. The goal of the sample was for extrapolation to generalized hospital populations. ECG reports used in the study came from across the health system: Outpatient Surgery, 


\section{Cureus}

Emergency Department, Med-Surgical Floors, Post-Anesthesia Care Units, the Intensive Care Unit, etc.

\section{QTc analysis}

QTcB was calculated using Phillips IntelliSpace ECG devices (Phillips Healthcare). QTcFri was calculated using the Fridericia formula (Table 1).

\begin{tabular}{|l|lll|}
\hline Creator & QTcX & Year & Formula \\
\hline Bazett [9] & QTcB & 1920 & QTc $=$ QT/JRR \\
Fridericia [10] & QTcFri & 1920 & QTc $=$ QT/3/JR \\
Hodges [11] & QTcH & 1983 & QTc $=$ QT + 1.75(HR-60) \\
Frammingham [12] & QTcFra & 1992 & QTc $=$ QT + 0.154(1-RR) \\
Dmitrienke [13] & QTcD & 2005 & QTc $=$ QT/RR ${ }^{0.413}$ \\
Rautaharju [6] & QTcF & 2014 & QTc $=$ QT - 185 (HR/60 - 1) + k (k = 6 ms [male], 0 ms [female $])$ \\
\hline
\end{tabular}

\section{TABLE 1: Common QTc formulae from 1920 - 2014}

QTc - Rate corrected QT interval, QT - Q wave to T wave interval, RR - R wave to R wave interval, HR - heart rate

Data were analyzed according to gender and for the aggregate total study population. General statistical methods included range, median, mean, and standard deviation. Confidence intervals were assessed to maintain the fidelity of analysis. The normality of data distribution was assessed with Kolmogorov-Smirnov testing. The Wilcoxon rank-sum test was then performed to confirm a statistically significant difference between the Bazett and Fridericia formulae. A value of $p<0.05$ was interpreted as significant. Statistical analysis was performed using SPSS statistical software (IBM Statistics, v. 26; IBM Corp, Armonk, NY).

QT interval rate correction was calculated by the Epic EHR and documented on each ECG report. Data were then stratified based on the presence of prolonged QTc, 450 and $460 \mathrm{~ms}$ standard cutoffs for men and women, respectively, and QTc greater than $500 \mathrm{~ms}$ - indicating greater risk for ventricular arrhythmia. The differences between the two formulae and their magnitudes were recorded along with respective means, standard deviations, and confidence intervals.

\section{Results}

\section{General characteristics}

A total of 61,946 ECG tracings were performed from May 2019 to April 2020 and uploaded to the Epic EHR at Cape Fear Valley Medical Center. After selecting the tracings that met the inclusion criteria, 44,566 ECGs were evaluated in this study (Table 2). 


\section{Cureus}

\begin{tabular}{|c|c|c|c|c|c|c|c|}
\hline \multirow{2}{*}{ Men $(n=19160)$} & \multirow[b]{2}{*}{ Mean } & \multirow[b]{2}{*}{ Standard Deviation } & \multirow[b]{2}{*}{ Minimum } & \multirow[b]{2}{*}{ Maximum } & \multirow[b]{2}{*}{ Median } & \multicolumn{2}{|l|}{$99 \% \mathrm{Cl}$} \\
\hline & & & & & & Lower & Upper \\
\hline Age (years) & 57 & 16.3 & 18 & 103 & 59 & 57.0 & 57.6 \\
\hline Heart Rate (bpm) & 83 & 14.6 & 61 & 120 & 82 & 83.2 & 87.7 \\
\hline QRS Duration (ms) & 95 & 10.6 & 49 & 119 & 94 & 94.6 & 95.0 \\
\hline QT Interval (ms) & 383 & 40.8 & 232 & 698 & 381 & 383.1 & 384.7 \\
\hline QTc Bazett (ms) & 448 & 37.9 & 303 & 749 & 444 & 447.7 & 449.1 \\
\hline QTc Fridericia (ms) & 425 & 35.3 & 277 & 730 & 421 & 424.8 & 426.1 \\
\hline$\Delta \mathrm{QTc}(\mathrm{ms})$ & 23 & 12.8 & 1 & 78 & 22 & 22.8 & 23.2 \\
\hline \multicolumn{8}{|l|}{ Women $(n=25406)$} \\
\hline Age (years) & 57 & 18.3 & 18 & 105 & 58 & 56.7 & 57.3 \\
\hline Heart Rate (bpm) & 83 & 14.6 & 60 & 120 & 83 & 82.7 & 83.2 \\
\hline QRS Duration (ms) & 91 & 10.6 & 43 & 119 & 91 & 91.1 & 91.5 \\
\hline QT Interval (ms) & 388 & 42.3 & 183 & 695 & 386 & 388.2 & 389.6 \\
\hline QTc Bazett (ms) & 453 & 37.6 & 209 & 738 & 448 & 452.0 & 453.2 \\
\hline QTc Fridericia (ms) & 430 & 35.7 & 200 & 716 & 425 & 429.3 & 430.5 \\
\hline$\Delta \mathrm{QTc}(\mathrm{ms})$ & 22.7 & 12.9 & 0 & 72 & 22 & 22.5 & 22.9 \\
\hline \multicolumn{8}{|l|}{ Total $(n=44566)$} \\
\hline Age (years) & 58 & 17.5 & 18 & 105 & 59 & 56.9 & $5 / .3$ \\
\hline Heart Rate (bpm) & 83 & 14.7 & 60 & 120 & 81 & 83.0 & 83.3 \\
\hline QRS Duration (ms) & 92.8 & 10.7 & 43 & 119 & 92 & 92.7 & 92.9 \\
\hline QT Interval (ms) & 387 & 41.7 & 183 & 698 & 384 & 386.2 & 387.2 \\
\hline QTc Bazett (ms) & 451 & 37.8 & 209 & 749 & 446 & 450.3 & 451.3 \\
\hline QTc Fridericia (ms) & 428 & 35.6 & 200 & 730 & 423 & 427.5 & 428.4 \\
\hline$\Delta \mathrm{QTc}(\mathrm{ms})$ & 22.8 & 12.9 & 0 & 78 & 22 & 22.7 & 23.0 \\
\hline
\end{tabular}

TABLE 2: ECG data obtained at Cape Fear Valley Medical Center from May 2019 to April 2020

ECG: electrocardiogram

The data showed $57 \%$ of the tracings were performed on females and the average age for both genders was $57 \pm 17.5$ years. The mean heart rate was $83 \pm 14.7 \mathrm{bpm}$ and the study population distribution is shown in Figure 1. 


\section{Cureus}

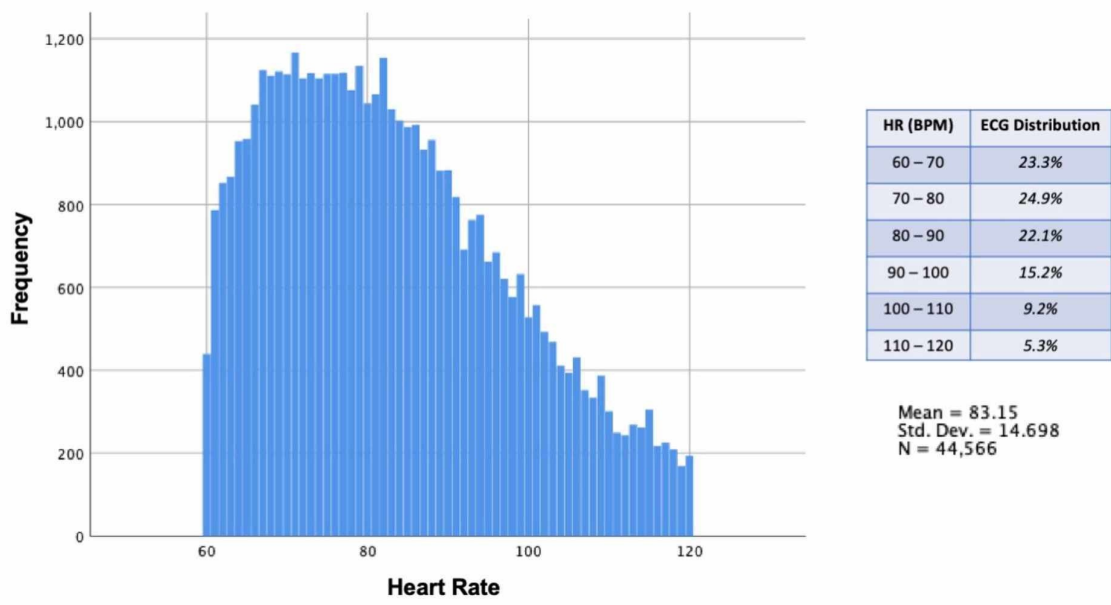

\section{FIGURE 1: Electrocardiogram report heart rate distribution}

${ }^{*}$ Created with IBM SPSS statistical software (IBM Corp., Armonk, NY)

The mean difference between the Bazett and Fridericia formulae for the total study population demonstrated a decrease in calculated QTc of $23 \pm 12.9 \mathrm{~ms}$ (99\% CI: $22.7,23.0 \mathrm{~ms}$ ). These data show minimal variation between sexes with mean age, heart rate, uncorrected QT, QTcB, QTcFri, and $\Delta$ QTc varying by less than $2 \%$. Mean QRSd showed the most variation between sexes with male QRSd $95 \pm 10.6 \mathrm{~ms}$. ( $99 \%$ CI: $94.6 \mathrm{~ms}, 95.0$ $\mathrm{ms}$ ) and female QRSd $91 \pm 10.6 \mathrm{~ms}$. (99\% CI: $91.1 \mathrm{~ms}, 91.5 \mathrm{~ms}$ ).

\section{QTc formula comparison}

Nonparametric distribution of the data was confirmed by Kolmogorov-Smirnov testing: $\mathrm{D}_{\text {Bazett }}(44,566)=$ $0.076, \mathrm{p}=<0.0001$ and $\mathrm{D}_{\text {Fridericia }}(44,566)=0.081, \mathrm{p}=<0.0001$.

Wilcoxon rank-sum testing (Table 3) of the difference (QTcB - QTcFri) revealed 44,127 ranks with a negative difference, 0 ranks with a positive difference, and 439 ties, p $<0.001$ (99\% CI: $22.5 \mathrm{~ms}, 23.0 \mathrm{~ms}$ ).

Wilcoxon Rank Sum Test QTcB-QTcF

$\begin{array}{lll}\mathrm{n} & 44,127 & \text { Negative Difference: } 44,127 \\ \mathrm{p} & <0.001 & \text { Positive Difference: } 0 \\ \text { Difference } & 22.5 \mathrm{~ms} & \text { Tie: } 439\end{array}$

$99 \% \mathrm{Cl}(\mathrm{ms}) \quad 22.5,23.0$

TABLE 3: QTc comparison statistical analysis

\section{Prolonged QTc group analysis}

QT interval prolongation criteria were met in 37.4\% of ECG reports (Table 4). Male ECG reports showed an increased incidence of QT interval prolongation but less frequent incidence than females at severely prolonged QTC > $500 \mathrm{~ms}$. Prolonged QT intervals corrected to the normal QT interval in $56.2 \%$ of cases with QTcFri (Table 4). 


\section{Cureus}

\begin{tabular}{|l|lllllll|l|}
\hline & \multicolumn{2}{l}{ Prolonged QTcB } & \multicolumn{2}{l}{ Prolonged QTcFri } & Corrected to Normal via QTcFri & $\Delta$ QTc (ms)[(99\% Cl) \\
\hline Women $(>460 \mathrm{~ms})$ & $34.20 \%$ & $8684 / 25406$ & $14.60 \%$ & $3714 / 25406$ & $57.20 \%$ & $4970 / 8684$ & $27.5(27.2,27.9)$ \\
Men $(>450 \mathrm{~ms})$ & $41.70 \%$ & $7981 / 19160$ & $18.70 \%$ & $3580 / 19160$ & $55.10 \%$ & $4401 / 7981$ & $27.7(27.3,28.1)$ \\
Total & $37.40 \%$ & $16665 / 44566$ & $19.60 \%$ & $8754 / 44566$ & $56.20 \%$ & $9371 / 16665$ & $27.6(27.3,27.9)$ \\
Mean $(\mathrm{ms})(99 \% \mathrm{Cl})$ & $487(486,488)$ & $459(459,460)$ & - & - & $27.6(27.3,27.9)$ \\
Median $(\mathrm{ms})$ & 477 & 452 & - & - & 28 \\
Standard Deviation $(\mathrm{ms})$ & 33.7 & 34.5 & - & - & 13.2 \\
\hline
\end{tabular}

TABLE 4: Prolonged QTC subgroup analysis

Utilizing QTcFri in place of QTcB decreased the corrected QT interval duration misinterpretation by $21 \%$ or 9,371 cases. The mean decrease in corrected QT interval duration was $28 \pm 13.2 \mathrm{~ms}$ ( $99 \%$ CI: $27.3 \mathrm{~ms}, 27.9$ $\mathrm{ms})$.

Table 5 shows that QTcFri use reduced the number of ECG reports with QTc $>500 \mathrm{~ms}$ by $57.3 \%$. A total of 125 ECG reports, 117 females and eight males, demonstrated correction to normal gender-specific QTc with QTcFri. The mean decreased in QTc with the Fridericia formula when QTcB $>500 \mathrm{~ms}$ was $31 \pm 14.5 \mathrm{~ms}(99 \%$ CI: 30.4, 31.7).

\begin{tabular}{|c|c|c|c|c|c|c|c|}
\hline \multirow[b]{2}{*}{ Women } & \multicolumn{2}{|c|}{ QTcB >500 ms } & \multicolumn{2}{|c|}{ QTcFri >500 ms } & \multicolumn{2}{|c|}{ Corrected to QTcFri $<500 \mathrm{~ms}$} & \multirow{2}{*}{$\begin{array}{l}\Delta \mathrm{QTc}(\mathrm{ms})(99 \% \mathrm{Cl} \\
30.6(29.8,31.4)\end{array}$} \\
\hline & $8.60 \%$ & $2185 / 25406$ & $3.80 \%$ & $975 / 25406$ & $55.30 \%$ & $1210 / 2185$ & \\
\hline Men & $7.70 \%$ & $1465 / 19160$ & $3.50 \%$ & $665 / 19160$ & $54.60 \%$ & $801 / 1465$ & $31.8(30.8,32.7)$ \\
\hline Total & $8.20 \%$ & $3650 / 44566$ & $3.50 \%$ & $1559 / 44566$ & $57.30 \%$ & $2901 / 3650$ & $31.0(30.4,31.7)$ \\
\hline Mean (ms) (99\% Cl) & \multicolumn{2}{|c|}{$536(535,538)$} & \multicolumn{2}{|c|}{$505(504,507)$} & - & - & $31.0(30.4,31.7)$ \\
\hline Median (ms) & 523 & & 494 & & - & - & 32 \\
\hline Standard Deviation (ms) & 38.3 & & 41.1 & & - & - & 14.5 \\
\hline
\end{tabular}

TABLE 5: Prolonged QTc > 500 ms subgroup analysis

\section{Discussion}

\section{Study design}

This study was undertaken to demonstrate the availability of evidence-directed QTc formulae on ECG reports without additional calculation. The Fridericia formula was not chosen as an alternative for the Bazett formula on the Epic EHR system, but QTcFri was programmed in the ECG report algorithm when the EHR was installed in May 2019. ECG reports were accessed for the parameters above which constituted the majority of the clinically relevant information found on said reports. The ECG reports did not comment on the computer interpretation of ECG rhythm or diagnosis.

This study did not take heart rhythm into account, QT-interval prolonging medications, electrolyte abnormalities present at the time of the electrocardiographic study, or medical history. Inclusion criteria were created to minimize the impact of these unknown factors. The physiologic heart rate without prolonged interventricular conduction delay was selected as a key criterion to remove, as much as possible, data interference from bradyarrhythmias and tachyarrhythmias.

\section{Main findings}

The results of the Wilcoxon rank-sum analysis, with a p-value of $<0.001$, confirms a statistically significant difference between the two formulae. In examining 44,566 ECG reports, when a difference existed, the magnitude was on average $22 \mathrm{~ms}$ but extended up to a maximum of $78 \mathrm{~ms}$. This analysis of rate-corrected QT evaluation using two power function formulae corroborates the 2009 American Heart Association 
(AHA)/American College of Cardiology Foundation (ACCF)/Heart Rhythm Society (HRS) recommendation to avoid QTCB use.

The greatest benefit of utilizing non- $\mathrm{QTcB}$ rate correction was evident when looking at the prolonged and severely prolonged QTc subgroups. The 16,665 ECG reports with prolonged QTcB corrected to normal QTc in $56 \%$ of cases when the Fridericia formula was applied. Evaluation of the 44,566 ECG reports in our health system over a one-year period showed $21 \%$ of the total ECGs corrected to normal QTc with evidence-based formulae. Inaccurately documenting one in five patient test results in the medical record is compelling evidence to change the standard of measurement.

The Fridericia formula is a power function; therefore, it is not endorsed by the AHA/ACCF/HRS guidelines. While QTcFri shows greater rate dependency than the Rautaharju formula, QTcFri also shows less rate dependency than the Bazett formula [2]. The Fridericia formula has been demonstrated in research to have a clinical benefit at physiologic heart rates [5]. QTcFri is the only calculation besides the Bazett formula mentioned by name in the US Food and Drug Administration's guidance for clinical trial evaluation of medication-induced QT interval prolongation [14].

\section{Clinical implications}

QT interval correction affects clinical decision-making. This data presents the opportunity to better educate providers on accurate methods to assess the QT interval. Viskin et al. (2005) demonstrated that the nonarrhythmia specialists (General Cardiologists, Internists, Neurologists, Pediatricians, Emergency Medicine Physicians, Intensivists, and Gastroenterologists) are not well-trained in measuring QT intervals, calculating QT intervals, or identifying QT interval prolongation [15]. Many providers are also not aware of which formula is used on ECGs or that multiple formulae exist. Studies like Viskin et al. (2005), in conjunction with the data above, demonstrate the clinical need for improved education to improve patient care [15].

When the QTc on ECG is interpreted as prolonged, therapies are potentially withheld or discontinued. This area of research holds promise for improving patient care: regarding the patient length of stay and nosocomial infection risk. With the demand for the use of QT-prolonging medications currently on the rise, utilizing more accurate data in the treatment of patients is needed.

Currently, the Epic EHR possesses the functionality to calculate QTcFri based on uploaded ECG data and to compare this calculation with ECG-documented QTcB data on each ECG report. This EHR capability creates the potential to increase provider awareness of evidence-based QTc formula and their integration into clinical practice.

\section{Limitations and future research}

This project was a single-center, non-randomized, non-blinded, retrospective, observational analysis, all of which has the potential to bias the data and subsequent results. These factors must be taken into consideration when evaluating the data.

ECG rhythm could not be determined from Epic ECG reports. The presence of atrial fibrillation, atrial flutter, and ventricular arrhythmia could not be assessed. Physiologic heart rate (60-120 bpm) was selected as an inclusion criterion to minimize the impact of arrhythmias on the fidelity of the data. The exclusion extent of irregular rhythms cannot be confirmed without viewing each of the 44,566 ECG tracings. Also, screening of cardiovascular disease history or QT-prolonging medication administration was not performed to allow a heterogeneous, real-world evaluation of patients independent of presentation.

Lastly, the fact that EHR systems possess the functionality to encode additional formulae like Fridericia into ECG reports presents the opportunity to employ guideline-based QTc functions like Hodges' formula (1983) or Rautaharju formula (2014) - both linear functions endorsed by the AHA/ACCF/HRS. The QTcR formula also has the added benefit of gender specificity evaluation for risk of TdP, with an adjusted QTc threshold for increased risk for ventricular arrhythmia at $477 \mathrm{~ms}$ [16]. Recent research like Rabkin et al.

(2015) demonstrates that the evaluation of this data and prospective studies with linear-function formulae may provide greater accuracy and further clinical benefit [2].

\section{Conclusions}

EHR QTc analysis with QTcFri demonstrated a mean decrease of $22 \mathrm{~ms}$ over 44,566 ECG tracings obtained at a single center between May 2019 and April 2020. During this time, 9,371 (21\%) of ECGs performed in a tertiary care facility were inaccurately documented with prolonged QTc. Accurate QT interval calculation will decrease the inappropriately documented test results and better inform downstream clinical decisionmaking through a wider scope of therapeutic intervention. This analysis is readily available to clinicians without any calculation required, and awareness of its existence will improve patient care. Just as the Fridericia formula was programmed in our EHR, the most accurate and guideline-directed QTc formulae are able to be employed. The utilization of this resource will provide the most accurate and clinically relevant data to inform clinical decision-making. EHR capability to program evidence-based QTc formulae without 
provider computation also merits further research to aid in the establishment of a new standard of QT rate correction.

\section{Additional Information \\ Disclosures}

Human subjects: Consent was obtained by all participants in this study. Cape Fear Valley Health Institutional Review Board issued approval N/A. The study was evaluated and deemed not to require oversight by the Cape Fear Valley Health Institutional Review Board and participant informed consent was waived as all data was collected retrospectively and deidentified. Animal subjects: All authors have confirmed that this study did not involve animal subjects or tissue. Conflicts of interest: In compliance with the ICMJE uniform disclosure form, all authors declare the following: Payment/services info: All authors have declared that no financial support was received from any organization for the submitted work. Financial relationships: All authors have declared that they have no financial relationships at present or within the previous three years with any organizations that might have an interest in the submitted work. Other relationships: All authors have declared that there are no other relationships or activities that could appear to have influenced the submitted work.

\section{Acknowledgements}

The authors gratefully acknowledge the statistical guidance from Dr. Riley Bowers of the Campbell University, School of Pharmacy.

\section{References}

1. Hurst JW: Naming of the waves in the ECG, with a brief account of their genesis . Circulation. 1998, 98:19371942. 10.1161/01.cir.98.18.1937

2. Rabkin SW, Cheng XB: Nomenclature, categorization and usage of formulae to adjust QT interval for heart rate. World J Cardiol. 2015, 7:315-325. 10.4330/wjc.v7.i6.315

3. Rautaharju PM, Surawicz B, \& Gettes LS: AHA/ACCF/HRS recommendations for the standardization and interpretation of the electrocardiogram: part IV: the ST segment, T and U waves, and the QT interval a scientific statement from the American Heart Association Electrocardiography and Arrhythmias Committee, Council on Clinical Cardiology; the American College of Cardiology Foundation; and the Heart Rhythm Society endorsed by the International Society for Computerized Electrocardiology. J Am Coll Cardiol. 2009, 53:982-991. 10.1016/j.jacc.2008.12.014

4. Sandau KE, Funk M, Auerbach A, et al.: Update to practice standards for electrocardiographic monitoring in hospital settings: a scientific statement from the American Heart Association. Circulation. 2017, 136:e273e344. 10.1161/cir.0000000000000527

5. Vandenberk B, Vandael E, Robyns T, et al.: Which QT correction formulae to use for QT monitoring? . J Am Heart Assoc. 2016, 5:e003264. 10.1161/JAHA.116.003264

6. Rautaharju PM, Mason JW, Akiyama T: New age- and sex-specific criteria for QT prolongation based on rate correction formulas that minimize bias at the upper normal limits. Int J Cardiol. 2014, 174:535-540. 10.1016/j.ijcard.2014.04.133

7. Lester RM, Paglialunga S, Johnson IA: QT assessment in early drug development: the long and the short of it. Int J Mol Sci. 2019, 20:1324. 10.3390/ijms20061324

8. Al-Khatib SM, LaPointe NMA, Kramer JM, Califf RM: What clinicians should know about the QT interval . JAMA. 2003, 289:2120-2127. 10.1001/jama.289.16.2120

9. Bazett HC: An analysis of the time-relations of electrocardiograms. Ann Noninvasive Electrocardiol. 2006, 2:177-194. 10.1111/j.1542-474x.1997.tb00325.x

10. Fridericia LS: Die Systolendauer im Elektrokardiogramm bei normalen Menschen und bei Herzkranken . Acta Med Scand. 1920, 53:469-486. 10.1111/j.0954-6820.1920.tb18266.x

11. Hodges M, Salerno D, Erlien D: Bazett's QT correction reviewed: evidence that a linear QT correction for heart rate is better. J Am Coll Cardiol. 1983, 1:694.

12. Sagie A, Larson MG, Goldberg R, et al.: An improved method for adjusting the QT interval for heart rate (the Framingham Heart Study). Am J Cardiol. 1992, 70:797-801. 10.1016/0002-9149(92)90562-d

13. Dmitrienke AA, Sides GD, Winter KJ, et al.: Electrocardiogram reference ranges derived from a standardized clinical trial population. Drug Inf J. 2005, 39:395-405. 10.1177/009286150503900408

14. Guidance for industry E14 clinical evaluation of QT/QTc interval prolongation and proarrhythmic potential for non-antiarrhythmic drugs. (2012). Accessed: June 28, 2020: https://www.fda.gov/media/71372/download.

15. Viskin S, Rosovski U, Sands AJ, et al.: Inaccurate electrocardiographic interpretation of long QT: The majority of physicians cannot recognize a long QT when they see one. Heart Rhythm. 2005, 2:569-574. 10.1016/j.hrthm.2005.02.011

16. Othong R, Wattanasansomboon S, Kruutsaha T, Chesson D, Vallibhakara SAO, Kazzi Z: Utility of QT interval corrected by Rautaharju method to predict drug-induced torsade de pointes. Clin Toxicol. 2018, 57:234-239. 10.1080/15563650.2018.1510501 